\title{
Applications of Atomic Force Microscopy in Industrial Polymer Systems \\ Lanti
}

Yang, Olivier Guise

SABIC Innovative Plastics, Department Technology and Innovation (T\&I), Bergen op Zoom, the Netherlands

In the polymer industry, scanning electron microscopy (SEM) and transmission electron microscopy (TEM) are commonly used techniques for material bulk and surface morphology characterization with nanoscale resolution [1]. Although both techniques have been widely used in practice we still find multiple limitations with these techniques for polymer morphology characterization: TEM sample preparation by cryo-microtoming, for example, is quite complex and time-consuming. In addition as the TEM image contrast is based on the staining procedure, it can offer a limited picture of the material for a complete morphology study of different phases in a polymer system. Another limitation is related to the electron beam damage or charging of the polymer material during both SEM and TEM characterization (especially with lower end SEMs where low voltage imaging is a challenge) and the difficulty related to $3 \mathrm{D}$ imaging of material surfaces with conventional equipment.

Atomic force microscopy (AFM) is a very useful technique with nano-meter scale resolution that allows the simultaneous characterization of polymer morphology and surface properties. With the development of AFM tapping mode height and phase imaging, AFM has emerged as a key microscopy tool for the characterization of the morphology of multiphase polymer systems and polymer blends [2]. In this abstract, we highlight the development and the benefits of morphology study by AFM on variety of polymer materials.

We successfully applied AFM for the morphology characterization of variety of key commercial resins containing hard and soft impact modifier (IM) components in the polymer blends. Specifically AFM offered significant safety (no staining step) and productivity (no thin-sectioning step) benefits over TEM while matching its resolution for the complete morphology characterization of polycarbonate (PC)/Styrene-acrylonitrile (SAN)/Acrylonitrile-butadiene-styrene (ABS) rubber materials (figure 1). Results show that AFM tapping mode phase imaging provides high contrast morphology images of such multiphase polymer systems. The morphology study using AFM gives us more opportunity and flexibility to characterize the polymer morphology along different flow directions, in the bulk and at the surface of the materials. These morphology studies enhance our understanding of the relationship between material structures and properties (optical, mechanical).

In the case of PC/Polybutylene terephthalate (PBT) polymer blends containing saturated IMs AFM - in combination with TEM - provides a complete morphology evaluation of the material. TEM imaging provides via differential staining high contrast between the PC and PBT phases. However, as saturated IMs cannot be stained with the common staining techniques, they typically appear as white particles in the PBT phase in TEM images (figure 2). AFM phase imaging provides a significant improvement in the contrast between the IM and the PC/PBT matrix. The high contrast of IM in the AFM images allows us to perform quantitative analysis of IM size distribution to further understand the influence of processing conditions on the material morphology and properties (figure 2). The results suggest that the combination of TEM and AFM techniques yields more complete morphology information of polymer 
systems containing saturated IM. This information is critical to further develop the properties of materials by dialing into specific processing conditions.

AFM has also been used with great success for challenging applications such as the characterization and quantification of the surface morphology of nano-scale porous membranes.

References:

[1] Sawyer, L. C.; et al., Polymer Microscopy Third Edition, Springer, 2008.

[2] Schonherr, H.; Vancso, G. J.; Scanning Force Microscopy of Polymers, Springer, 2010.

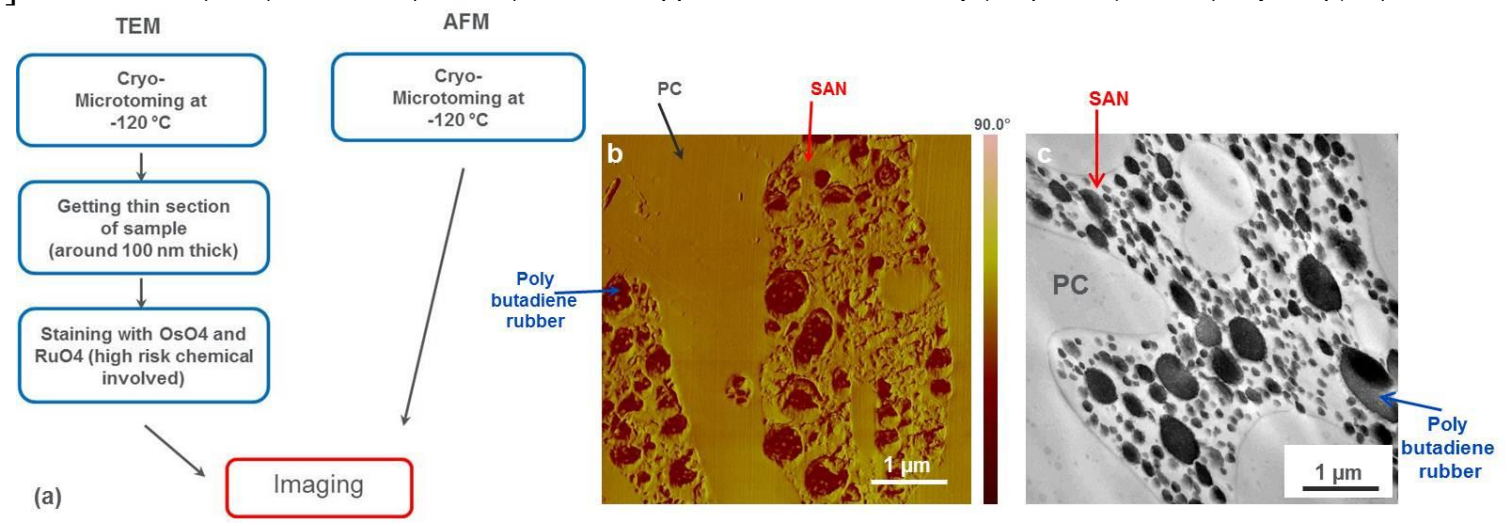

Figure 1. (a) TEM and AFM sample preparation procedures for general impact modifier contained polymer system. (b) AFM phase images and (c) TEM image of polymer blends (PC/SAN/ABS HRG). Different components as indicated in the images: PC is the continuous phase, SAN is in the PC matrix, and polybutadiene rubber is dispersed in SAN.
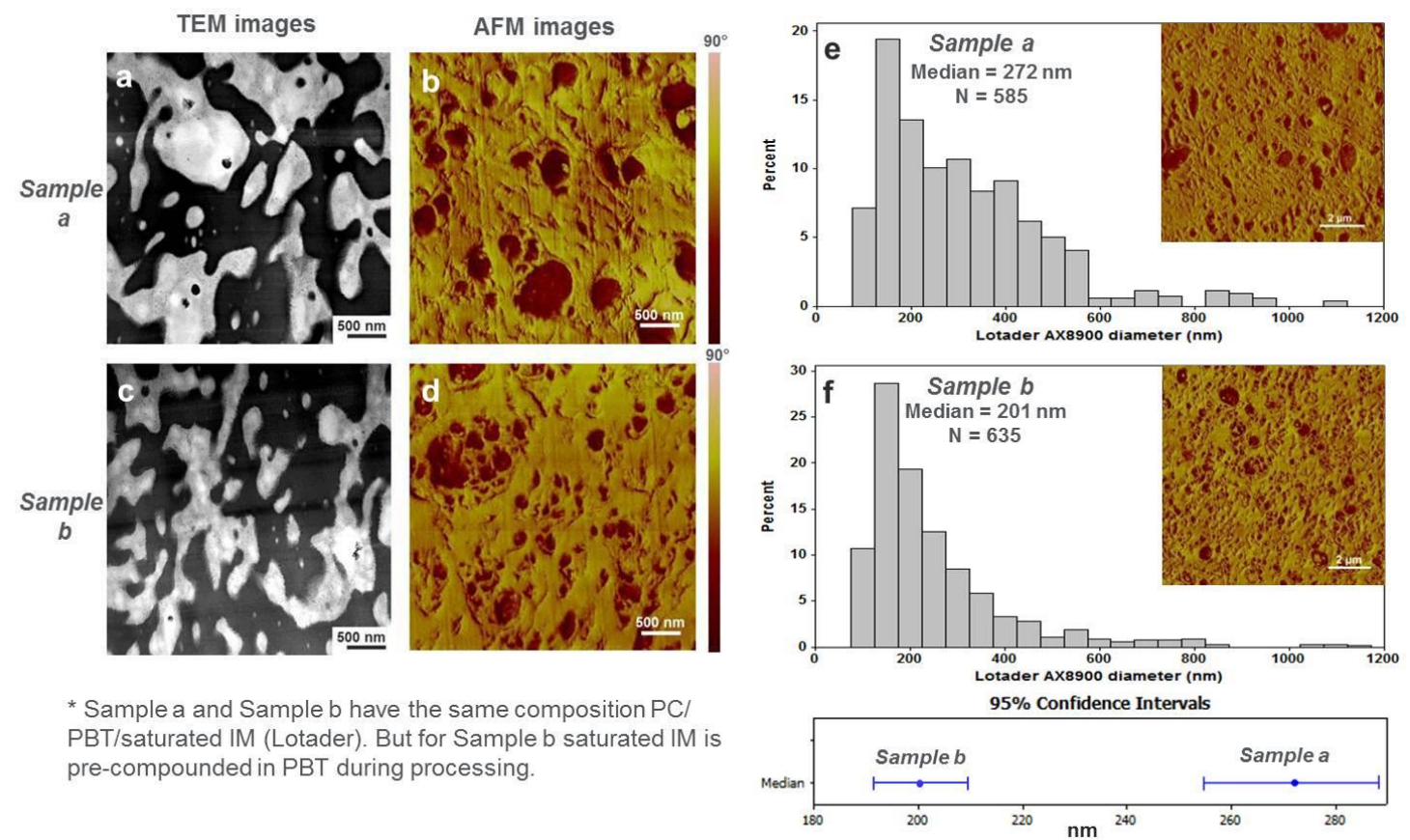

Figure 2. TEM ( $\mathrm{a} \& \mathrm{c}$ ) and AFM (b \& d) phase images of PC/PBT blends containing saturated IM. TEM images show the PC/PBT matrix but the saturated IM Lotader $†$ shows unclear contrast. AFM phase images show the morphology of Lotader $\dagger$ IM as darker phase in the image. (e) and (f) Lotader $\dagger$ IM domain size distribution and median particle size of sample a and sample b. Typical AFM phase images of the analyzed area were also included in the figures. 